\title{
Repères
}

\section{Colloques et documents : comptes rendus}

\author{
« BiodiverCities 2015 - Villes et parcs naturels : construire une nature urbaine ?» \\ (Colloque, Marseille, 17-19 septembre 2015)
}

Dans le contexte d'un intérêt croissant pour la nature en ville, la série de colloques BiodiverCities, organisée essentiellement par des géographes, a proposé de porter une attention particulière aux espaces protégés urbains et périurbains pour offrir des éléments de réponse à une question simple mais cruciale : "Urbanisation et protection sont-elles antagonistes ou complémentaires?». L'édition 2015 de ce colloque, quatrième du genre (après Paris 2010, Rio de Janeiro 2012 et Le Cap 2014), s'est tenue à Marseille, dans les locaux de l'École supérieure du professorat et de l'éducation (ESPE), les 17 et 18 septembre, et sur le terrain dans les Calanques, le 19 septembre. L'intitulé «Villes et parcs naturels : construire une nature urbaine? » invitait à s'intéresser particulièrement aux multiples imbrications entre l'espace urbain et l'espace protégé, entre espaces verts et espaces protégés (plus largement que les seuls «parcs naturels »), au risque d'un amalgame récurrent entre différentes formes de nature urbaine.

Le partage d'expériences en termes de gestion des parcs naturels urbains et périurbains, la présentation des recherches actuelles sur les interactions conservation/ développement, sociétés/nature et villes/espaces naturels, et l'impulsion d'une coopération entre les gestionnaires des espaces protégés de différents pays du Sud étaient les trois grands objectifs fixés par ce colloque. Le colloque a accueilli environ 150 personnes de tout horizon, dont des étudiants de l'École nationale supérieure d'architecture de Marseille (ENSA-M) et d'Aix-Marseille Université.

\section{Un dialogue en construction entre gestionnaires, responsables et scientifiques}

Les colloques BiodiverCities se veulent des rencontres entre scientifiques, gestionnaires et responsables de plusieurs parcs nationaux, organisées par le réseau UPA (Urban Protected Areas) et le programme ANR UNPEC (Urban National Parks in Emerging Countries and Cities). Cette édition fut élaborée en partenariat avec les
Parcs nationaux des Calanques et de Port-Cros - Porquerolles et la ville de Marseille ${ }^{1}$.

Quatre tables rondes étaient composées essentiellement des différents responsables et gestionnaires de parcs des mégapoles concernées par le programme UNPEC (Nairobi [Kenya], 3 millions d'habitants ; Le Cap [Afrique du Sud], 2,5 millions ; Mumbai [Inde], 20 millions et Rio de Janeiro [Brésil], 12 millions) auxquels s'ajoutaient des intervenants des parcs nationaux français partenaires susmentionnés. Entre ces tables rondes se sont tenues quatre sessions scientifiques de communications, sélectionnées par le comité scientifique : 16 sur 32 propositions ont été retenues. Au sein de celles-ci, le fort tropisme méridional s'est exprimé avec trois communications sur le Parc national des Calanques; les autres concernaient le Parc national Nevado de Toluca (Mexique, deux interventions), des cas australien, suisse, finlandais, mais aussi les villes de Dehli, Belo Horizonte, Pau et du Val de Loire.

Ce type d'organisation a permis avec succès d'exposer les principes de gestion auprès des scientifiques et, en retour, de faire entendre les analyses des spécialistes des espaces protégés urbains auprès des gestionnaires et responsables.

En revanche, il est quelque peu regrettable que la majorité des discussions des tables rondes n'aient pas été plus abouties, laissant percevoir une sorte de consensus mou entre des acteurs pourtant exposés à des contextes et des problématiques très variés. Beaucoup se sont en effet abrités derrière une rhétorique de la bonne gouvernance souvent creuse et peu argumentée, alors qu'il aurait été préférable d'entendre les réels problèmes auxquels gestionnaires et responsables doivent répondre, dans leur diversité et leur complexité. Certaines tables rondes se résumaient davantage à une succession de présentations (dont certaines reprenaient des éléments factuels à propos des parcs déjà énoncés) sans véritable

\footnotetext{
La présentation et le programme du colloque sont disponibles en ligne à l'adresse http:/ / www.upa-network.org/.
} 
échange ni interaction. La table ronde 2 «Quelle place de la ville dans l'agenda des gestionnaires de parcs nationaux? ", en confrontant les avis des directeurs de parcs nationaux, a sans doute été celle où la mise en perspective a été la plus intéressante. En outre, la dernière journée sur le terrain a su pallier ces manquements en abordant très concrètement les enjeux actuels auxquels le Parc national des Calanques doit faire face (voir plus loin).

\section{Une réelle acuité des recherches scientifiques sur les espaces protégés urbains}

La bonne qualité globale des communications a permis de soulever de réelles interrogations et de proposer de véritables analyses. Un bémol toutefois, avant de détailler les quatre sessions scientifiques, l'annulation de quatre des seize interventions prévues initialement.

Dès l'introduction du colloque, Frédéric Landy (responsable du programme UNPEC, professeur en géographie à l'Université Paris Ouest Nanterre La Défense) a évoqué les grandes problématiques transversales à l'ensemble des espaces protégés urbains et proposé une grille d'analyse pertinente. Ainsi, pour comparer les différents terrains d'études et appréhender les tensions entre politiques de conservation et développement économique et social, entre ville et nature, entre besoins locaux et logiques urbaines mondialisées, il a suggéré de se référer à un double gradient d'intégration et d'interaction ville/parc, d'une part, des jeux d'échelles traduisant une mondialisation plus ou moins effective des espaces protégés, d'autre part. Les quatre parcs nationaux particulièrement étudiés dans le cadre du programme UNPEC peuvent ainsi être différenciés à l'aune des logiques et des stratégies actorielles. En conséquence, alors que les Parcs nationaux de la montagne de la Table au Cap et de Tijuca à Rio de Janeiro sont fortement intégrés à la ville et à l'espace mondialisé, celui de Nairobi est partie prenante des logiques mondialisées sans être intégré aux dynamiques urbaines locales ; quant au Parc national Sanjay Gandhi à Mumbai, il demeure en marge de l'espace urbain et de la mondialisation. L'avis n'est, on s'en doute, pas partagé par les gestionnaires de l'espace protégé en question, mais l'analyse a permis de problématiser en amont les présentations des parcs nationaux parties prenantes du programme, parfois trop linéaires et descriptives.

\section{Flux, frontières et connectivités}

La première session, consacrée aux limites entre l'espace protégé et la ville, et aux échanges qui s'opèrent entre ces deux entités, a d'abord montré la difficulté de sortir $\mathrm{du}$ périmètre spatial et du cadre réglementaire $\mathrm{du}$ parc pour s'inscrire plus largement dans une logique urbaine, au-delà des simples «fronts » qui sont les formes les plus fréquentes du contact entre ville et espace protégé. Ces fronts ne se limitent pas à des barrières et à une confrontation rigide ; ils ont une véritable épaisseur et sont plastiques, mouvants, marqués par la porosité.

Les deux premières communications (Cécilia Claeys et al. [Université d'Aix-Marseille] ; Stéphane Héritier et Clotilde Lebreton [Université Jean-Monnet-SaintÉtienne et École nationale supérieure de Lyon]), l'une à propos des usages et des controverses environnementales du Parc national des Calanques et l'autre relative à la recatégorisation du Parc national Nevado de Toluca au Mexique (déclassé de son statut de parc national en 2013 pour devenir une aire de protection de la flore et de la faune), ont suggéré une forte imbrication de l'espace protégé et de l'espace urbain par les pratiques qui s'y déploient. Si cette imbrication s'observe dans bien d'autres cas, sa traduction administrative et réglementaire est loin d'être évidente. L'équilibre est souvent délicat entre une protection forte, excluante, faisant des espaces protégés des exclaves écosystémiques, et une protection faible, servant de faire-valoir à la préservation d'espaces verts urbains. Dépasser les logiques aréolaires et de juxtaposition pour penser en termes réticulaires et d'imbrication permettrait de mieux intégrer les parcs naturels à l'espace urbain. La dernière communication (Miguel Giorgeff et al.), assurée par une équipe du Parc national des Calanques, évoquait l'élaboration d'entités paysagères destinées à devenir des unités de gestion au sein desquelles la concertation s'est engagée de 2014 à 2016. Elle posait la question des opérations de renaturation des espaces dégradés, mais était plus à la marge de la session, en avançant davantage une réflexion sur la naturalité de l'espace protégé que sur les limites ville/ parc national.

\section{Usages et usagers, restrictions et accès à la nature et à la ville}

La deuxième session ne comprenait que deux communications relevant d'horizons très différents. L'une (Claudia M. Orduz Rojas et al. [Université fédérale du Minas Gerais, Brésil]) portait sur les conflits à propos de la création du Parc national de Gandarela, au sein de l'aire métropolitaine de Belo Horizonte (Brésil), où les jeux d'acteurs habituels sont inversés. Ce sont en effet certaines populations locales qui sont les plus demandeuses d'une politique de conservation qui, paradoxalement, leur permettrait de faire perdurer leurs activités extractives. La seconde communication (Amélie Robert et Jean-Louis Yengué [Université de Tours]) concluait à partir d'une étude dans la région Centre que les citadins étaient prêts à accepter davantage de nature en ville, à condition que les espaces naturels soient entretenus. Elle était clairement hors sujet puisqu'elle traitait des espaces verts et non d'espaces protégés urbains, ce qui illustre la confusion encore persistante entre les deux. Les deux 
communications, en dépit de leurs approches méthodologiques et leurs contextes sociopolitiques très différents, ont traduit la difficulté pour les gestionnaires de mettre en place des politiques de protection ou d'écologisation en adéquation avec les attentes citoyennes.

\section{Construire une nature urbaine}

Dans la troisième session, quatre communications (Sylvie Clarimont et Kildine Leichnig [Université de Pau et des Pays de 1'Adour] ; Camille Girault [Université Savoie Mont Blanc] ; Joëlle Salomon Cavin [Université de Lausanne] ; Megha Sud [Université de Cologne]) ont abordé, dans des contextes toujours très distincts (France, Finlande, Suisse, Inde), les différentes conceptions de la nature et de la ville, et par conséquent de la nature urbaine telle que produite par les politiques de protection. Les valeurs écologiques, sociales et économiques afférentes aux espaces protégés expliquent en partie les difficultés de gestion entre volonté de protection forte ou meilleure acceptation d'une protection faible. L'exemple du « parc naturel urbain » palois (statut auto-créé et diffusé sous la forme d'une association, qui n'est guère repris ailleurs en France et qui ne rencontre plus guère de succès depuis le changement de municipalité en 2014) montre que l'appellation peut davantage être utilisée à des fins de marketing urbain, sans qu'elle soit accompagnée d'un contenu réglementaire contraignant. Entre les exemples suisse, finlandais, français et indien, une grande disparité de dispositifs de protection existe. Leurs divers degrés et leurs finalités plurielles, les logiques nationales et locales singulières rendent difficilement transposables des formes de préservation pensées dans une ville, pour une ville, par les acteurs d'une ville. Au-delà de ces exemples particuliers, un regard croisé sur ces communications permet de dégager trois éléments saillants, autour des valeurs et des affichages, de l'outillage de la protection (quels moyens pour la conduire ?) et des rapports entre urbanité et nature ; l'ensemble soulignait l'intensité variable de l'intégration des espaces protégés dans la ville et de l'urbain dans les politiques de protection.

\section{La protection au prisme des échelles}

La quatrième session proposait trois communications (Anne Cadoret et Xavier Daumalin [Université Aix-Marseille] ; Samuel Depraz et al. [Université Lyon 3] ; Adrienne Keane [Université de Sidney]) de nouveau éclectiques par leurs terrains d'études (encore une fois les Calanques et l'ex-parc Nevado de Toluca au Mexique, mais aussi l'Australie) et par leurs approches. Cependant, toutes se rejoignaient sur un point, à savoir la nécessité de comprendre comment la planification urbaine entre dans la gestion des espaces protégés urbains et, inversement, comment les politiques de protection influencent les documents d'urbanisme. Il a ainsi été souligné l'importance de la prise en compte des héritages antérieurs à l'instauration d'un espace protégé et du contexte spatial dans lequel il s'inscrit. De la sorte, les marges de la ville et les limites de l'espace protégé deviennent des portes d'entrée de l'une vers l'autre, des lieux à part entière riches de nombreux échanges. Les conflits, endogènes et exogènes, la volonté de prise de contrôle du foncier et de privatisation des espaces font partie des éléments remarquables de ces communications.

\section{Une sortie sur le terrain au cœur des problématiques du Parc national des Calanques (19 septembre 2015)}

La sortie, conduite le samedi en guise de clôture du colloque, a permis à une quarantaine de personnes de revenir sur la mise en place de ce jeune parc national (avril 2012), dont l'équipe se constitue progressivement. Il s'agissait de gagner depuis la porte de Luminy le belvédère de Sugiton (altitude de $248 \mathrm{~m}$ ), entre la calanque éponyme et celle de Morgiou.

\section{La complexité de gestion d'un parc national périurbain et fortement fréquenté}

La large palette de propriétaires fonciers (villes de Marseille et de Cassis, conseil général, ministère de la Défense, etc.) et la grande proximité urbaine expliquent les difficultés de gestion, par exemple, la prévention des incendies de forêts : le domaine de Luminy a brûlé 3 ou 4 fois depuis 30 ans. La porte de Luminy, une des six entrées du parc national, accueille 10000 personnes par jour durant l'été. La calanque de Sugiton, la plus accessible depuis cette porte, compte jusqu'à 5000 personnes par jour sur ses deux plages, avec des incidences fortes sur la végétation.

En périphérie de Marseille, le site de Luminy a été acquis par l'État dans les années 1960 afin de construire un complexe sportif et un des campus de l'Université d'Aix-Marseille (qui rapatrie néanmoins aujourd'hui certaines de ses fonctions universitaires en plein centreville, comme l'ENSA-M). Près de 200 kilomètres de sentiers mis en place dès la fin du XIX ${ }^{\mathrm{e}}$ siècle par la Société des excursionnistes marseillais et le Club alpin français (CAF) sillonnent la partie calanques du parc national. $\mathrm{Au}$ moment de la création du groupement d'intérêt public pour la mise en place du parc national, il y a douze ans, les Calanques comptaient 2000 voies d'escalade ; elles en comptent désormais près du double, les grimpeurs s'étant activés pour en ouvrir de nouvelles avant la création du parc national. Cela a conduit à de fortes tensions entre le parc et la Fédération française de la montagne et de l'escalade (FFME). 


\section{Des usages du cœur contraires aux objectifs et aux caractéristiques d'un parc national ?}

Comme les autres parcs nationaux, les Calanques comptent un cœur (ici terrestre et marin), ex-zone centrale où s'applique la réglementation, et une aire d'adhésion (ex-zone périphérique) que rejoignent les communes après le vote de leur conseil municipal, et où les acteurs s'accordent sur des « orientations ». Le cœur terrestre de ce parc national est caractérisé par de nombreux équipements : la route départementale D559 du col de la Gineste, la voie SNCF entre Cassis et La Ciotat et deux kilomètres de l'autoroute A50. La ville ceinturant littéralement le cœur de parc, l'aire d'adhésion sur Marseille et sur certaines communes limitrophes est réduite à une étroite bande. Par exemple, le vignoble de Cassis, le deuxième en France à obtenir une AOC en 1936, a été intégré dans l'aire d'adhésion afin d'accentuer la conversion vers le bio (un exemple $\mathrm{d}^{\prime}$ « orientation » possible).

Les précédents sites classés (celui des Calanques en 1975 et celui du cap Canaille en 1980) n'ont pas permis d'aboutir à une protection satisfaisante. Sans grande richesse faunistique (à part un couple d'aigles de Bonelli) et floristique (espèces des éboulis calcaires méditerranéens), ce sont les paysages des Calanques qui ont justifié la création du parc national : Sugiton, En-Vau et Port-Pin pour les non-bâties, Morgiou et Sormiou pour celles rendues célèbres par leurs " cabanons », rattachés à un «village» (un des 110 quartiers de Marseille). Ces deux dernières calanques sont alimentées en électricité, mais pas en eau (réserves sur les toits). Morgiou est équipée du téléphone, ce qui n'est pas le cas de Sormiou ; cette dernière bénéficie d'une vidange de $90 \%$ des « eaux noires » de ses cabanons, alors que les acteurs de la première n'ont pas résolu le problème et évacuent les eaux en Méditerranée.

\section{La mise en place poussive de la réglementation}

En cœur marin du parc, les activités nautiques (jet-ski, ski nautique, bouée tractée) sont interdites. Une réserve intégrale, telle que permise par la loi de 2006 sur les parcs nationaux dans leurs cœurs, est en projet au droit des zones de non-prélèvement (interdites de pêche), mais sa faisabilité est incertaine au vu des difficultés de gestation des deux seules existantes depuis la loi de 1960 (lac de Lauvitel, parc national des Écrins [1995] et îlots de PortCros [2007]). Les infractions sont plus nombreuses en mer (quatre à dix par jour en été) que sur terre (une tous les quinze jours durant la belle saison). À défaut d'avoir une équipe de gardes-moniteurs étoffée, le parc national fonctionne avec des jeunes recrutés comme éco-gardes qui œuvrent pour la sensibilisation des visiteurs. En effet, son budget est actuellement de trois millions d'euros et ses effectifs de trente personnes. La réglementation se met ainsi en place progressivement : un décret voté par le conseil d'administration et mis en œuvre par le directeur concernera le ramassage des plantes. Autre défi de taille dans les années qui viennent, l'interdiction en cœur de parc de la pêche amateur qui alimente de manière «parallèle » la restauration marseillaise à hauteur de $100 \mathrm{~kg}$ par jour.

\section{Le défi constant du traitement des eaux usées de l'agglomération}

Jusqu'en 1986, les eaux de l'Huveaune étaient rejetées telles quelles à proximité des plages $\mathrm{du} 8^{\mathrm{e}}$ arrondissement de Marseille. Elles ont été détournées, sont traitées dans une station d'épuration et rejetées au large de la calanque de Cortiou. Toutefois, en cas de fortes précipitations, le trop-plein d'eau emprunte le cours initial et la station d'épuration de l'agglomération, pourtant refaite en 2000, ne peut traiter les volumes d'effluents lors de ces forts débits. Actuellement, un gigantesque chantier de canalisations est financé dans le cadre du plan d'action du contrat de baie ( 280 millions d'euros sur 5 ans), à proximité du stade Vélodrome pour évacuer ce tropplein d'eau de manière satisfaisante. Il en va de l'ouverture administrative des plages de la ville de Marseille.

\section{Les Calanques, produit d'appel touristique international}

De surcroît, la pression est forte du côté des décideurs de la ville et de l'agglomération de Marseille (devenue métropole d'Aix-Marseille-Provence au $1^{\mathrm{er}}$ janvier 2016) qui se sont dotées d'une ambition touristique croissante : la cité phocéenne recevait 15000 croisiéristes il y a 20 ans et en a enregistré 1,5 million en 2015, devenant la troisième escale méditerranéenne. Les élus comptent en faire d'ici quelques années la deuxième mondiale derrière Miami. Ces escales apportent près de 15000 visiteurs à la journée, voire 30000 le week-end, qui sillonnent la ville. Les perspectives pour les tour-opérateurs de vendre une visite maritime ou terrestre dans les Calanques au cours de l'escale font craindre aux gestionnaires du parc national un afflux touristique non contrôlé. En effet, sur les 26000 ha de la commune (une des plus vastes de France), près de 9000 ha sont des espaces naturels - aux niveaux de naturalité très variables, comme indiqué plus haut. Parmi eux, les Calanques, sur lesquelles comptent jouer les acteurs municipaux. Elles sont déjà très demandées pour des tournages de séries ou de films, obligeant les équipes du parc national à un suivi serré de leurs conditions de réalisation, alors que les décideurs municipaux incitent à cette valorisation lucrative.

\section{Un colloque riche de perspectives}

Ce colloque a indéniablement été très riche ; il a permis de soulever des problématiques transversales aux 
espaces protégés urbains, bien au-delà des contextes d'urbanisation et de développement très différents. En conclusion, Frédéric Landy a alerté sur le risque de confusion entre parc national et ville durable, qui sous-tendait certaines interventions. Les échanges d'expériences entre les responsables et les gestionnaires des différents parcs nationaux urbains des pays émergents impulsés par le programme UNPEC ont trouvé ici une sorte de point d'orgue ; les acteurs des Parcs nationaux de Port-Cros et des Calanques ont à la fois nourri le débat et pu entendre des contributions faisant écho à leurs préoccupations quotidiennes; quant aux chercheurs, ils ont su à juste titre nuancer certaines postures, développer des problématiques spécifiques aux espaces protégés urbains et d'autres génériques sur les enjeux des sociétés contemporaines majoritairement urbaines, et favoriser le dialogue à toutes les échelles. Le programme UNPEC sera sans doute prolongé sous une autre forme (séminaires, publications...) à partir de 2017, notamment dans le cadre de la décennie des Nations unies pour la biodiversité.

Camille Girault et Lionel Laslaz (Université Savoie Mont Blanc, UMR EDYTEM, Le Bourget-du-Lac, France) camille.girault@univ-savoie.fr lionel.laslaz@univ-savoie.fr 